\title{
General and Specific Combining Ability Analysis for Yield and Some of Its Associated Characters in Late Sown Condition in Bread Wheat (Triticum aestivum L.em. Thell)
}

\author{
Ritu Choudhary*, Hoshiyar Singh, Pawan Kumar and Sheetal Raj Sharma \\ Division of Plant Breeding and Genetics, Rajasthan Agricultural Research Institute, \\ Durgapura, SKNAU, Jobner \\ *Corresponding author
}

\section{A B S T R A C T}

In order to analyze combining ability for yield and some of its associated characters in late sown condition in bread wheat (Triticum aestivum L.em. Thell), the present experiment was carried out performing diallel analysis following Griffing (1956) Method II Model I. Ten genetically diverse parents were selected and crossed in half diallel fashion excluding

\begin{tabular}{|l|}
\hline K e y w or d s \\
General and \\
specific combining \\
ability, Yield, \\
Bread wheat
\end{tabular}
reciprocals. These parents were evaluated along with their 45 F1's and 45 F2's in a randomized block design with 3 replications. Significant differences were observed among the parents, generations, F1, F2 and parents v/s generations for all the characters except for number of tillers per plant among the parents and grain filling period for parents v/s generations. Significant mean squares due to GCA and SCA for all the characters except GCA for number of tillers per plant in F1 and SCA for number of tillers per plant in F1 indicating that both additive and non-additive gene effects played an important role in the genetic control of the traits studied. The GCA / SCA variance ratio was less than unity indicated the importance of non-additive gene action for all the characters under investigation. Based on an overall evaluation of GCA effects and per se performance, the parent parents Raj 3765, Raj 4083 and Raj 4079 emerged as good general combiners while the crosses Raj 4238 X Raj 4238 and Raj 4120 X DBW 17 emerged as good specific cross combinations for grain yield and some other attributes.. As a consequence of predominant non-additive gene action in the present study, breeding approaches such as reciprocal recurrent selection, diallel selective mating, bi-parental mating and multiple cross would be useful breeding approaches for developing new genotypes from potent breeding material identified.

\section{Introduction}

Wheat, a cereal crop which stands at prime position among cereals in terms of acreage, production and versatility in adaptation to wider range of agro-environmental situations.
Wheat is the third most produced food grain in the world after maize and rice which stands second to rice as the main human feed and ahead of maize as maize is more extensively used for animal feed. It is staple food for more than one billion people of the country and also 
in as many as 43 countries of the world. In India even after the making the noteworthy progress in wheat production during recent years, it is necessary to further more increase the productivity level of the crop to meet the requirements and to keep pace with the burgeoning population growing at $1.2 \%$ per annum (Anonymous, 2015) and changing food habits of rural and urban population. Hence, the fundamental objective of most of the plant breeding programmes remain to evolve varieties with improved and high yield potential. Yield which is a complex character influenced by various contributing traits, sowing time, environmental stresses and so. (confirmed by various studies and Grafius (1959) even doubted the existence of genes for yielding ability) Hence. Improvement in yield requires component breeding approach. For this, a breeder needs to identify suitable breeding methodology, which primarily depends upon the combining ability, nature and magnitude of different types of gene actions and overall inheritance pattern for yield and yield contributing characters.

Diallel mating design is widely used in plant breeding research to obtain information on genetic effects for a fixed set of parental lines or estimates of general combining ability, specific combining ability.

Therefore, using ten genetically diverse parents viz., Raj 3765, DBW 621 50, Raj 3077, Raj 4079, Raj 4120, HD 2967, Raj 4083, Raj 4238, DBW 17 and DBW 88 wwre subjected to diallel mating (excluding reciprocals) in the present study.

\section{Materials and Methods}

On the basis of genetic diversity and their stability for different yield traits, ten diverse wheat varieties selected as the experimental material and crossed in diallel mating fashion excluding reciprocals to obtain $45 \mathrm{~F} 1 \mathrm{~s}$ in rabi
2014-2015. In Rabi 2015-16 ten genotypes along with their $45 \mathrm{~F} 1$ 's and $45 \mathrm{~F} 2$ 's (obtained at IARI, regional station, Wellington, TN during kharif, 2015) progenies were evaluated using Randomized Block in 3 replications at Agricultural Research Farm of RARI, Durgapura.

Observations were recorded on days to heading (Days), days to maturity (Days), grain filling period (Days), plant height (cm.), flag leaf area $(\mathrm{cm} 2)$, number of tillers per plant, spike length $(\mathrm{cm})$, number of grains per spike, 1000-seed weight $(\mathrm{g})$, harvest index $(\%)$ and grain yield/plant (g). Mean values over selected plants were used for statistical analysis. The mean values of different parents, F1s and F2s for all characters were subjected for analysis of variance and the estimates of variance for general combining ability and specific combining ability and their effects were computed by Method 2 (Model I) as suggested by Griffing (1956).

\section{Results and Discussion}

Analysis of variance revealed significant differences among parents except for number of effective tillers per plants, significant differences were also observed among the genotypes and generations for all the characters. Further analysis revealed significant mean squares due to parents and F1 progenies and $\mathrm{F} 2$ progenies. This is also in conformity with the findings obtained by Kaukab et al., (2014), Samir and Ismail (2015), Bhardwaj (2017) and Yadav (2017).

Mean squares due to F1 vs F2 were found significant for all the characters. Similarly, the differences among parents vs generation were significant for all the traits except for grain filling period indicated the presence of heterosis in all the three environments. Yada (2017) reported similar results (Table 1-6). 
The combining ability analysis revealed significant mean squares due to GCA and SCA for all the characters in F1 and F2 generations except GCA for number of tillers per plant in F1. The significant differences of GCA and SCA indicated that both additive and non-additive gene effects played an important role in the genetic control of the traits under study. These findings were in close agreement with those reported by Adel et. al. (2013), Kalhoro et. al. (2015). Kumar and Kerkhi (2015), Mari et al., (2015) and Samir and Ismail (2015), Bhardwaj (2017) and Yadav (2017).

The GCA / SCA variance ratio was less than unity indicated the importance of non-additive gene action for all the characters under investigation. The results were in conformity with findings obtained by Irshad et al., (2014), Kaukab et al., (2014), Mari et al., (2015), Kumar et al., (2015), Bhardwaj (2017) and Yadav (2017). The preponderance of nonadditive genetic variance for most of the characters indicated that the best cross combinations might be selected on the basis of SCA for further tangible advancement in wheat.

Consistency for desirable GCA estimates for more than one specific or many characters, three parents namely; Raj 3765, Raj 4083 and Raj 4079 were good general combiners. Therefore, these parents have good potential and may be used in synthesizing a dynamic population with most of the favorable genes accumulated.

In self-pollinated crops like wheat, SCA effects have relatively less applicability as they are consequences of non-additive gene effects except those arising from complementary gene action or linkage effects and cannot be fixed in the end product i.e. pure line. Jinks and Jones (1958) emphasized that the superiority of the hybrids might not indicate their ability to yield transgressive segregants, rather SCA would provide satisfactory criteria. However, if a cross combination exhibs high SCA as well as high per se performance having at least one parent as good general combiner for a specific trait, it is expected that this cross combination may provide desirable transgressive segregants in later generations.

In the present study, none of the crosses showed consistently high SCA effects in desirable direction for all the characters. However, based on the relative consistent exhibition of high SCA effects in desirable direction for yield and some of its contributing characters the crosses Raj 3765 X Raj 4238 and Raj 4120 X DBW 17 appeared as good specific cross combinations for grain yield and some associated traits.

The parents Raj 3765 was good general combiners for grain yield and one or two yield contributing traits while the other parents were emerged as average or poor combiners. Further, both additive and non-additive gene action were operating for most of the characters, thus recurrent selection is an appropriate choice (Hull, 1945), but it is often not very effective with partial dominance (Moll et. al., 1964). The additive gene action has been exploited more in wheat as being a self pollinated crop, whereas the non-additive variance which is outcome of dominance and epistasis gene interaction remains to be utilized, which can be exploited for further improvement of wheat through systematic breeding programme for the targeted environment. Overall appraisal of the results in the present study, advocated that reciprocal recurrent selection (Hull, 1945) diallel selective mating, use of multiple crosses and bi-parental mating may be effective alternative approaches for tangible advancement of bread wheat yield in the coming years. 
Table.1 Analysis of variance sowing mean squares for parents $F_{1}$ 's and $F_{2}$ 's for yield and its contributing attributes

\begin{tabular}{|c|c|c|c|c|c|c|c|c|c|c|c|c|}
\hline & df & $\begin{array}{l}\text { Days to } \\
\text { heading }\end{array}$ & $\begin{array}{l}\text { Days to } \\
\text { maturity }\end{array}$ & $\begin{array}{l}\text { Grain } \\
\text { filling } \\
\text { period }\end{array}$ & $\begin{array}{l}\text { Plant } \\
\text { height }\end{array}$ & $\begin{array}{l}\text { Flag } \\
\text { leaf } \\
\text { area }\end{array}$ & $\begin{array}{l}\text { Effective } \\
\text { tiller per } \\
\text { plant }\end{array}$ & $\begin{array}{l}\text { Spike } \\
\text { length }\end{array}$ & $\begin{array}{l}\text { Grain } \\
\text { per } \\
\text { spike }\end{array}$ & $\begin{array}{l}1000 \\
\text { grain } \\
\text { weight }\end{array}$ & $\begin{array}{l}\text { Harvest } \\
\text { index }\end{array}$ & $\begin{array}{l}\text { Grain } \\
\text { yield per } \\
\text { plant }\end{array}$ \\
\hline Replications & 2 & 0.46 & 10.59 & 0.35 & 8.54 & 13.17 & 1.31 & 1.84 & 3.20 & 2.17 & 3.95 & 9.45 \\
\hline genotypes & 99 & $20.11 * *$ & $48.44 * *$ & $74.29 * *$ & $64.22 * *$ & $19.74 * *$ & $2.67 * *$ & $10.06 * *$ & $97.38 * *$ & $69.77 * *$ & $46.26 * *$ & $33.82 * *$ \\
\hline parents & 9 & $6.96 * *$ & $33.94 * *$ & $61.36 * *$ & $79.59 * *$ & $23.50 * *$ & 0.58 & $12.20 * *$ & $67.52 * *$ & $19.18 * *$ & $34.29 * *$ & $66.19 * *$ \\
\hline generations & 89 & $21.36 * *$ & $50.00 * *$ & $76.42 * *$ & $62.37 * *$ & $19.18 * *$ & $2.78 * *$ & $9.67 * *$ & $93.49 * *$ & $70.53 * *$ & $47.50 * *$ & $29.64 * *$ \\
\hline $\mathbf{F}_{1}$ & 44 & $17.57 * *$ & $62.28 * *$ & $70.39 * *$ & $59.16 * *$ & $17.59 * *$ & $2.15^{* *}$ & $8.54 * *$ & $88.45 * *$ & $72.35 * *$ & $36.57 * *$ & $20.99 * *$ \\
\hline $\mathbf{F}_{2}$ & 44 & $18.46^{* *}$ & $38.24 * *$ & $72.76^{* *}$ & $64.46 * *$ & $20.25 * *$ & $1.96 * *$ & $7.97 * *$ & $100.11 * *$ & $67.23 * *$ & $54.50 * *$ & $19.34 * *$ \\
\hline $\mathbf{F}_{1} \mathrm{vs}_{\mathbf{2}} \mathbf{F}_{2}$ & 1 & $316.07 * *$ & $26.70 *$ & $503.15^{* *}$ & $111.38 * *$ & $42.49 * *$ & $66.61 * *$ & $134.73 * *$ & $23.79 *$ & $135.76 * *$ & $220.75 * *$ & $863.42 * *$ \\
\hline $\begin{array}{l}\text { Parents vs } \\
\text { generations }\end{array}$ & 1 & $26.78 * *$ & $40.28 *$ & 1.25 & $90.25 * *$ & $35.14 *$ & $11.24 * *$ & $25.49 * *$ & $712.09 * *$ & $456.60 * *$ & $43.34 * *$ & $114.73 * *$ \\
\hline error & 198 & 1.89 & 6.04 & 1.72 & 5.37 & 5.97 & 1.03 & 1.44 & 4.60 & 3.99 & 4.27 & 3.90 \\
\hline
\end{tabular}

Table.2 Analysis of variance for general and specific combining ability for yield and its contributing attributes

\begin{tabular}{|c|c|c|c|c|c|c|c|c|c|c|c|c|c|}
\hline & & df & $\begin{array}{ll}\text { Days to } \\
\text { Heading }\end{array}$ & $\begin{array}{ll}\text { Days } & \text { to } \\
\text { Maturity }\end{array}$ & $\begin{array}{l}\text { Grain } \\
\text { Filling } \\
\text { Period }\end{array}$ & $\begin{array}{l}\text { Plant } \\
\text { Height } \\
\text { cm }\end{array}$ & $\begin{array}{l}\text { Flag } \\
\text { Leaf } \\
\text { Area } \mathrm{cm}^{2}\end{array}$ & $\begin{array}{l}\text { Tillers/ } \\
\text { Plant }\end{array}$ & $\begin{array}{l}\text { Spike } \\
\text { Length }\end{array}$ & $\begin{array}{l}\text { Grains/ } \\
\text { Spike }\end{array}$ & $\begin{array}{l}1000 \\
\text { grain } \\
\text { weight }\end{array}$ & $\begin{array}{l}\text { Harvest } \\
\text { Index } \\
(\%)\end{array}$ & $\begin{array}{l}\text { Grain } \\
\text { Yield/ } \\
\text { Plant (g) }\end{array}$ \\
\hline \multirow[t]{2}{*}{ GCA } & $\mathrm{F}_{1}$ & 9 & $10.67 * *$ & $32.92 * *$ & $17.99 * *$ & $44.20 * *$ & $12.32 * *$ & 0.56 & $5.64 * *$ & $43.20 * *$ & $5.66^{* *}$ & $16.10 * *$ & $35.88 * *$ \\
\hline & $\mathrm{F}_{2}$ & 9 & $11.98 * *$ & $19.82 * *$ & $16.64 * *$ & $55.23 * *$ & $13.63 * *$ & $0.62 *$ & $5.33 * *$ & $47.47 * *$ & $5.55 * *$ & $21.14 * *$ & $16.43 * *$ \\
\hline \multirow[t]{2}{*}{ SCA } & $\mathrm{F}_{1}$ & 45 & $4.06 * *$ & $16.13 * *$ & $23.68 * *$ & $16.86 * *$ & $4.94 * *$ & $0.63^{*}$ & $2.98 * *$ & $30.06 * *$ & $8.11 * *$ & $11.01 * *$ & $31.68 * *$ \\
\hline & $\mathrm{F}_{2}$ & 45 & $4.87 * *$ & $11.19 * *$ & $24.93 * *$ & $15.53 * *$ & $5.87 * *$ & $0.79 * *$ & $2.36^{* *}$ & $31.89 * *$ & $8.62 * *$ & $16.68 * *$ & $7.70 * *$ \\
\hline \multirow[t]{2}{*}{ Error } & $\mathrm{F}_{1}$ & 108 & 0.39 & 2.21 & 0.66 & 1.93 & 1.88 & 0.40 & 0.59 & 2.23 & 0.86 & 1.47 & 1.10 \\
\hline & $\mathrm{F}_{2}$ & 108 & 0.86 & 2.11 & 0.48 & 1.60 & $5.87 * * *$ & 0.31 & 0.42 & 1.35 & 1.50 & 1.38 & 1.42 \\
\hline
\end{tabular}


Table.3 Estimates of general combining ability effects

\begin{tabular}{|c|c|c|c|c|c|c|c|c|c|c|}
\hline & $\begin{array}{l}\text { Days to } \\
\text { Heading } \\
\text { E1F1 }\end{array}$ & $\begin{array}{l}\text { Days to } \\
\text { Heading } \\
\text { E1F2 }\end{array}$ & $\begin{array}{l}\text { Days to } \\
\text { Maturity } \\
\text { E1F1 }\end{array}$ & $\begin{array}{l}\text { Days to } \\
\text { Maturity } \\
\text { E1F2 }\end{array}$ & $\begin{array}{l}\text { Grain } \\
\text { Filling } \\
\text { Period } \\
\text { E1F1 }\end{array}$ & $\begin{array}{l}\text { Grain } \\
\text { Filling } \\
\text { Period } \\
\text { E1F2 }\end{array}$ & $\begin{array}{l}\text { Plant } \\
\text { Height } \\
\text { cm E1F1 }\end{array}$ & $\begin{array}{l}\text { Plant } \\
\text { Height } \\
\text { cm E1F2 }\end{array}$ & $\begin{array}{lr}\text { Flag } & \text { Leaf } \\
\text { Area } & \mathbf{c m}^{2} \\
\text { E1F1 } & \end{array}$ & $\begin{array}{ll}\text { Flag } & \text { Leaf } \\
\text { Area } & \mathbf{c m}^{2} \\
\text { E1F2 } & \end{array}$ \\
\hline Raj 3765 & $-0.38 *$ & -0.28 & $-1.45^{* * *}$ & -0.31 & 0.34 & $0.54 * *$ & $-1.49 * * *$ & $1.66 * * *$ & 0.29 & 0.56 \\
\hline DBW 62150 & $0.57 * *$ & $0.51 *$ & $1.99 * * *$ & $1.32 * *$ & $2.13 * * *$ & $1.94 * * *$ & $2.87 * * *$ & $3.84 * * *$ & $-1.39 * * *$ & $-1.33 * *$ \\
\hline Raj 3077 & $0.39 *$ & 0.39 & $1.25^{* *}$ & $1.22 * *$ & $-0.77 * * *$ & $-0.71 * * *$ & $3.09 * * *$ & $2.81 * * *$ & $-1.25 * *$ & $-1.35^{* * *}$ \\
\hline Raj 4079 & 0.51** & 0.64* & $1.55 * * *$ & $1.52 * * *$ & $0.82 * * *$ & $0.78 * * *$ & -0.56 & 0.13 & -0.65 & -0.65 \\
\hline Raj 4120 & $0.98 * * *$ & $0.78 * *$ & 0.64 & -0.31 & $-0.97 * * *$ & $-0.78^{* * *}$ & 0.63 & 0.57 & $-0.92 *$ & $-1.11 * *$ \\
\hline HD 2967 & $0.42 *$ & 0.49 & -0.42 & -0.23 & $-1.15 * * *$ & $-1.38 * * *$ & $-2.26 * * *$ & $-2.57 * * *$ & $1.75^{* * *}$ & $1.84 * * *$ \\
\hline Raj 4083 & $-2.27 * * *$ & $-2.56 * * *$ & $-3.23 * * *$ & $-2.36 * * *$ & $-0.84 * * *$ & $-0.93 * * *$ & $-1.64 * * *$ & $-1.84 * * *$ & 0.51 & 0.66 \\
\hline Raj 4238 & $-0.56^{* *}$ & $-0.67 *$ & $-1.59 * * *$ & $-0.95^{*}$ & $-1.27 * * *$ & $-1.24 * * *$ & -0.37 & -0.40 & 0.77* & $0.82 *$ \\
\hline DBW 17 & -0.30 & 0.28 & $1.02 *$ & $1.18^{* *}$ & $1.73^{* * *}$ & $1.53 * * *$ & $-1.62 * * *$ & $2.05 * * *$ & 0.66 & 0.37 \\
\hline DBW 88 & $0.64 * * *$ & 0.41 & 0.25 & $-1.09^{* *}$ & -0.02 & 0.25 & $1.36^{* * * *}$ & $1.17^{* *}$ & 0.23 & 0.19 \\
\hline \multicolumn{11}{|c|}{$\begin{array}{l}\text { CD Comparisons for } \\
\text { GCA }\end{array}$} \\
\hline $\mathrm{Gi}<>>0$ at $95 \%$ & 0.38 & 0.58 & 0.92 & 0.90 & $0.51 * * *$ & 0.43 & 0.86 & 0.78 & 0.85 & 0.89 \\
\hline $\mathrm{Gi} i>>$ at $99 \%$ & 0.55 & 0.83 & 1.32 & 1.29 & $0.73 * * *$ & 0.62 & 1.24 & 1.13 & 1.22 & 1.28 \\
\hline $\mathrm{Gi}-\mathrm{Gj}$ at $95 \%$ & 0.57 & 0.86 & 1.37 & 1.34 & $0.75 * * *$ & 0.64 & 1.28 & 1.17 & 1.27 & 1.33 \\
\hline $\mathbf{G i}-\mathbf{G j}$ at $99 \%$ & 0.82 & 1.23 & 1.97 & 1.93 & $1.08 * * *$ & 0.92 & 1.84 & 1.68 & 1.82 & 1.91 \\
\hline$h^{2}$ Narrow Sense & 0.30 & 0.28 & 0.24 & 0.21 & 0.11 & 0.10 & 0.29 & 0.37 & 0.26 & 0.25 \\
\hline$h^{2}$ Broad Sense & 0.93 & 0.87 & 0.90 & 0.85 & 0.97 & 0.98 & 0.92 & 0.93 & 0.72 & 0.73 \\
\hline GCA/SCA Ratio & 0.23 & 0.23 & 0.18 & 0.16 & 0.06 & 0.06 & 0.24 & 0.32 & 0.28 & 0.25 \\
\hline Predictability Ratio & 0.32 & 0.32 & 0.27 & 0.25 & 0.11 & 0.10 & 0.32 & 0.39 & 0.36 & 0.34 \\
\hline
\end{tabular}

Table.4 Estimates of general combining ability effects

\begin{tabular}{|c|c|c|c|c|c|c|c|c|c|c|c|c|}
\hline & $\begin{array}{r}\text { Tillers/ } \\
\text { Plant } \\
\text { E1F1 }\end{array}$ & $\begin{array}{l}\text { Tillers } \\
\text { Plant } \\
\text { E1F2 }\end{array}$ & $\begin{array}{r}\text { Spike } \\
\text { Length } \\
\text { E1F1 }\end{array}$ & $\begin{array}{r}\text { Spike } \\
\text { Length } \\
\text { E1F2 }\end{array}$ & $\begin{array}{r}\text { Grains/ } \\
\text { Spike } \\
\text { E1F1 }\end{array}$ & $\begin{array}{r}\text { Grains/ } \\
\text { Spike } \\
\text { E1F2 }\end{array}$ & $\begin{array}{r}\text { 1000- } \\
\text { grain } \\
\text { Weight } \\
\text { (g) E1F1 }\end{array}$ & $\begin{array}{r}\text { 1000- } \\
\text { grain } \\
\text { Weight } \\
\text { (g) E1F2 }\end{array}$ & $\begin{array}{r}\text { Harvest } \\
\text { Index } \\
\text { \%) E1F1 }\end{array}$ & $\begin{array}{r}\text { Harvest } \\
\text { Index } \\
\text { (\%) E1F2 }\end{array}$ & $\begin{array}{r}\text { Grain } \\
\text { Yield/ } \\
\text { Plant (g) } \\
\text { E1F1 }\end{array}$ & $\begin{array}{r}\text { Grain } \\
\text { Yield/ } \\
\text { Plant (g) } \\
\text { E1F2 }\end{array}$ \\
\hline Raj 3765 & 0.24 & $0.31 *$ & $0.96^{* * *}$ & $0.64 * * *$ & $4.06^{* * *}$ & $4.29 * * *$ & 0.55 & 0.64 & 1.90 *** & $1.88 * * *$ & $1.83 * * *$ & $1.35 * * *$ \\
\hline DBW 62150 & $-0.37 *$ & $-0.35 *$ & $-1.18^{* * * *}$ & $-0.98 * * *$ & $-1.28 * *$ & $-1.43 * * *$ & $-1.55 * * *$ & $-1.73 * * *$ & $-1.51 * * *$ & $-1.92 * * *$ & $-2.98 * * *$ & $-1.72 * * *$ \\
\hline Raj 3077 & -0.08 & -0.10 & -0.25 & -0.24 & $0.92 *$ & $0.65^{*}$ & -0.50 & $-0.65 *$ & $-1.29 * * *$ & $-1.33 * * *$ & $-0.64 *$ & $-0.94 * *$ \\
\hline Raj 4079 & 0.25 & 0.28 & $0.718^{* *}$ & $0.91 * * *$ & $0.84 *$ & 0.45 & $1.27 * * *$ & $1.27 * * *$ & $1.06^{* *}$ & $0.99 * *$ & $1.71 * * *$ & $1.55 * * *$ \\
\hline Raj 4120 & -0.30 & -0.26 & -0.15 & -0.19 & -0.25 & $0.95 * *$ & $-1.14 * * *$ & $-0.74 *$ & -0.54 & 0.38 & $-0.86 * *$ & 0.27 \\
\hline HD 2967 & 0.06 & 0.12 & 0.10 & 0.01 & $-2.37 * * *$ & $-3.34 * * *$ & $1.94 * * *$ & $2.13 * * *$ & $-0.76 *$ & $-2.03 * * *$ & -0.29 & $-0.95 * *$ \\
\hline Raj 4083 & 0.16 & 0.13 & $0.47 *$ & $0.91 * * *$ & 0.47 & $0.70 *$ & $1.12^{* * *}$ & $1.11^{* * *}$ & $1.35^{* * *}$ & 0.33 & $1.96^{* * *}$ & $1.15^{* * *}$ \\
\hline Raj 4238 & 0.04 & -0.03 & 0.18 & 0.17 & -0.16 & -0.38 & 0.36 & 0.38 & 0.25 & $0.80 *$ & $0.71 *$ & 0.01 \\
\hline DBW 17 & 0.14 & 0.11 & 0.17 & $-0.41 *$ & 0.36 & $-0.82 *$ & $-0.62 *$ & $-0.78 *$ & 0.29 & $0.94 * *$ & $0.90 * *$ & 0.55 \\
\hline DBW 88 & -0.14 & -0.22 & -1.01 & $-0.83 * * *$ & $-2.58 * * *$ & $-1.08 * * *$ & $-1.42 * * *$ & $-1.63 * * *$ & $-0.75 *$ & -0.05 & $-2.33 * * *$ & -1.28 \\
\hline \multicolumn{13}{|c|}{$\begin{array}{l}\text { CD Comparisons for } \\
\text { GCA }\end{array}$} \\
\hline $\mathrm{Gi} i>>0$ at $95 \%$ & 0.39 & 0.34 & 0.48 & 0.40 & 0.93 & 0.72 & 0.65 & 0.73 & 0.75 & 0.73 & 0.65 & 0.74 \\
\hline$G i<>0$ at $99 \%$ & 0.56 & 0.49 & 0.68 & 0.58 & 1.33 & 1.04 & 0.93 & 1.06 & 1.08 & 1.04 & 0.94 & 1.06 \\
\hline $\mathbf{G i}-\mathbf{G j}$ at $95 \%$ & 0.58 & 0.51 & 0.71 & 0.60 & 1.38 & 1.07 & 0.97 & 1.09 & 1.12 & 1.08 & 0.97 & 1.10 \\
\hline $\mathbf{G i}-\mathbf{G j}$ at $99 \%$ & 0.84 & 0.74 & 1.02 & 0.86 & 1.98 & 1.54 & 1.39 & 1.57 & 1.61 & 1.56 & 1.39 & 1.58 \\
\hline $\mathbf{h}^{2}$ Narrow Sense & 0.04 & 0.06 & 0.22 & 0.26 & 0.19 & 0.19 & 0.23 & 0.25 & 0.18 & 0.16 & 0.15 & 0.25 \\
\hline$h^{2}$ Broad Sense & 0.40 & 0.63 & 0.85 & 0.87 & 0.94 & 0.97 & 0.91 & 0.89 & 0.89 & 0.93 & 0.97 & 0.86 \\
\hline GCA/SCA Ratio & 0.06 & 0.05 & 0.18 & 0.21 & 0.12 & 0.13 & 0.17 & 0.19 & 0.13 & 0.11 & 0.09 & 0.20 \\
\hline Raj 3765 & 0.11 & 0.10 & 0.26 & 0.30 & 0.20 & 0.20 & 0.26 & 0.28 & 0.20 & 0.18 & 0.16 & 0.28 \\
\hline
\end{tabular}


Table.5 Estimates of specific combining ability

\begin{tabular}{|c|c|c|c|c|c|c|c|c|c|c|}
\hline & \multicolumn{2}{|c|}{ Days to Heading } & \multicolumn{2}{|c|}{ Days to Maturity } & \multicolumn{2}{|c|}{ Grain Filling Period } & \multicolumn{2}{|c|}{ Plant Height cm } & \multicolumn{2}{|c|}{ Flag Leaf Area $\mathrm{cm}^{2}$} \\
\hline & $\mathrm{F}_{1}$ & $\mathrm{~F}_{2}$ & $\mathrm{~F}_{1}$ & $F_{2}$ & $\mathrm{~F}_{1}$ & $\mathrm{~F}_{2}$ & $\mathrm{~F}_{1}$ & $\mathrm{~F}_{2}$ & $\mathrm{~F}_{1}$ & $\mathrm{~F}_{2}$ \\
\hline P1XP2 & -0.41 & -0.81 & $-4.33 * *$ & $-2.95^{*}$ & $-1.90 *$ & $-1.59 *$ & -0.27 & -1.22 & -2.37 & $-2.71 *$ \\
\hline P1XP3 & -0.98 & $-2.03 *$ & 0.41 & 0.81 & -0.12 & 0.10 & 1.34 & 1.53 & 1.29 & 1.44 \\
\hline P1XP4 & 0.48 & 0.06 & -1.90 & -1.16 & $-2.96^{* * *}$ & $-2.25 * *$ & $-4.65^{* * *}$ & $-5.00 * * *$ & $4.10 * *$ & $5.16^{* * *}$ \\
\hline P1XP5 & -0.61 & -1.24 & 2.02 & $4.01 * *$ & $-4.04 * * *$ & $-3.17 * * *$ & 0.58 & 1.67 & 0.00 & -0.42 \\
\hline P1XP6 & -0.42 & -0.46 & $-4.92 * * *$ & $-4.41 * *$ & $-9.20 * * * *$ & $-9.12 * * *$ & $-3.07 *$ & $-3.19 * *$ & 0.99 & 1.28 \\
\hline P1XP7 & -0.69 & -0.41 & 0.56 & 0.40 & $7.95^{* * * *}$ & $8.32 * * *$ & 0.29 & 0.20 & 0.88 & 0.45 \\
\hline P1XP8 & $-2.23 * * *$ & $-2.63 * *$ & -2.42 & -2.35 & $-2.57 * *$ & $-2.20 * *$ & $-4.84 * * *$ & $-4.10 * *$ & 2.11 & 1.45 \\
\hline P1XP9 & $3.33^{* * * *}$ & $2.31 * *$ & $3.96 * *$ & 1.51 & $-1.78^{*}$ & -0.79 & $6.69 * * *$ & $7.29 * * *$ & -0.71 & -0.37 \\
\hline P1XP10 & 0.37 & 0.92 & -0.92 & 0.12 & 1.09 & $2.40 * * *$ & $2.90 *$ & $3.18 * *$ & -2.06 & -2.30 \\
\hline P2XP3 & $-3.05 * * *$ & $-3.00 * *$ & $-3.36^{*}$ & -1.15 & $-1.69 *$ & $-1.53^{*}$ & -2.15 & $-2.79 *$ & $2.85^{*}$ & 2.54 \\
\hline P2XP4 & $3.45^{* * * *}$ & $2.14 *$ & 2.67 & 1.55 & $-5.60 * * *$ & $-5.26 * * *$ & $-7.16 * * *$ & 1.21 & 0.44 & 0.70 \\
\hline P2XP5 & -1.07 & -0.91 & 1.59 & 0.71 & $7.42 * * *$ & $7.85 * * *$ & 0.91 & 1.45 & -0.17 & -2.06 \\
\hline P2XP6 & $-1.37 *$ & -1.59 & 0.64 & 0.93 & $4.17 * * *$ & $5.58 * * *$ & $2.68 *$ & $2.74 *$ & 0.07 & -0.10 \\
\hline P2XP7 & -0.32 & -0.66 & $4.01 * *$ & $4.10 * *$ & $2.93 * * *$ & $3.98 * * *$ & $3.42 *$ & $4.43^{* * * *}$ & -1.57 & -0.13 \\
\hline P2XP8 & $1.56^{* *}$ & 1.57 & $5.81 * * *$ & $3.02 *$ & $3.47 * * *$ & $3.63 * * *$ & $2.89^{*}$ & $2.39 *$ & -1.06 & -1.30 \\
\hline P2XP9 & $-1.62 * *$ & $-2.38 * *$ & -1.14 & 1.14 & -0.71 & $-1.31^{*}$ & $-5.09 * * *$ & $-5.96 * * *$ & 0.04 & -0.08 \\
\hline P2XP10 & $3.35 * * *$ & $2.38 * *$ & $4.97 * * *$ & -0.18 & $7.64 * * *$ & $8.09 * * *$ & -0.34 & -1.38 & $-4.44 * *$ & $-4.15 * *$ \\
\hline P3XP4 & $-2.92 * * *$ & $-3.02 * * *$ & $-4.59 * *$ & $-4.35 * *$ & -1.33 & -0.46 & -0.80 & -1.51 & 0.67 & 0.42 \\
\hline P3XP5 & $1.28^{*}$ & 0.87 & 2.33 & -0.52 & 0.97 & 0.84 & 1.20 & 1.55 & -2.36 & -2.09 \\
\hline P3XP6 & $-1.15^{*}$ & -1.13 & -1.95 & $-3.93 * *$ & $2.38 * *$ & $2.52 * * *$ & -1.94 & -1.32 & -1.29 & -1.30 \\
\hline P3XP7 & $1.20^{*}$ & 0.92 & 1.53 & 1.20 & $1.90 *$ & $3.98 * * *$ & -1.22 & -1.21 & 1.29 & 1.22 \\
\hline P3XP8 & -0.85 & -1.30 & -0.45 & 0.45 & $-1.58^{*}$ & $-1.63^{*}$ & 1.32 & 1.60 & -0.26 & -0.23 \\
\hline P3XP9 & $1.89 * *$ & 0.60 & 1.94 & 0.32 & $-2.74 * * *$ & $-1.82 * *$ & 1.61 & 1.91 & -1.16 & -0.46 \\
\hline P3XP10 & 0.87 & 0.84 & 1.38 & $3.26^{*}$ & $3.13 * * *$ & $3.60 * * *$ & 1.30 & 1.88 & -1.13 & $-3.34 *$ \\
\hline P4XP5 & $2.16^{* * *}$ & $1.96^{*}$ & $5.02 * * *$ & 2.50 & $-3.68 * * *$ & $-3.97 * * *$ & $6.35^{* * *}$ & $5.55^{* * * *}$ & $-3.90 * *$ & $-3.40 *$ \\
\hline P4XP6 & -0.39 & -0.38 & -0.92 & 0.43 & 0.63 & $1.77 * *$ & -1.55 & -2.21 & -1.22 & $-2.99 *$ \\
\hline P4XP7 & $-1.26^{*}$ & -1.00 & $-7.11 * * *$ & $-6.10 * * *$ & -7.67 & $-6.68 * * *$ & $-3.86 * *$ & $-4.66 * * *$ & $3.23 *$ & 2.39 \\
\hline P4XP8 & 0.69 & 0.78 & -1.09 & -2.19 & 9.18 & $9.46 * * *$ & $-5.80 * * *$ & $-5.64 * * *$ & -0.86 & -0.93 \\
\hline P4XP9 & $4.44 * * *$ & $3.16^{* * *}$ & -2.37 & -0.98 & 2.38 & $1.97 * *$ & 2.41 & 2.10 & $-3.77 * *$ & $-3.66 * *$ \\
\hline P4XP10 & $-1.91 * *$ & $-1.96^{*}$ & $3.41^{*}$ & 2.29 & 0.43 & 1.14 & $2.83^{*}$ & $2.79 *$ & -0.71 & -1.03 \\
\hline P5XP6 & 0.88 & 0.82 & 1.66 & -0.74 & 1.47 & $2.11 * *$ & $4.38 * *$ & $4.25 * * *$ & -0.36 & -0.26 \\
\hline P5XP7 & -1.10 & -1.13 & $-7.53 * * *$ & $-6.60 * * *$ & 0.31 & 0.99 & $-7.07 * * *$ & $-7.45 * * *$ & 1.41 & 1.70 \\
\hline P5XP8 & 0.22 & 0.64 & -1.84 & -0.02 & $5.75 * * *$ & $6.36^{* * *}$ & -0.92 & -0.76 & 2.15 & $2.70^{*}$ \\
\hline P5XP9 & $-1.37 *$ & $-2.97 * *$ & $-8.45 * * *$ & $-5.48 * * *$ & $-9.86^{* * * *}$ & $-9.14 * * *$ & $-5.84 * * *$ & $-5.83 * * *$ & $3.02 *$ & 1.69 \\
\hline P5XP10 & $3.57 * * *$ & 1.57 & -2.01 & $-5.88 * * *$ & $-1.67^{*}$ & 0.02 & 1.33 & 2.29 & -2.23 & -2.35 \\
\hline P6XP7 & 0.84 & $-2.52 * *$ & 0.87 & 1.65 & $-2.05 * *$ & $-3.79 * * *$ & 1.47 & $2.44^{*}$ & -1.62 & -1.86 \\
\hline P6XP8 & -0.21 & $-4.81 * * *$ & $2.85^{*}$ & $4.23 * *$ & $2.67 * * *$ & $3.53 * * *$ & -1.24 & -0.75 & -0.89 & -0.89 \\
\hline P6XP9 & $-3.13 * * *$ & $2.94 * *$ & -2.39 & -1.57 & $-2.42 * *$ & $-2.24 * *$ & -0.98 & -0.99 & 0.55 & -0.14 \\
\hline P6XP10 & $2.92 * * *$ & $1.85^{*}$ & $6.05^{* * *}$ & $5.04 * * *$ & 1.35 & $2.18^{* *}$ & $4.10 * *$ & $3.77 * *$ & 0.31 & 1.88 \\
\hline P7XP8 & $-1.52 *$ & -0.69 & -2.30 & -2.30 & $-6.65 * * *$ & $-5.27 * * *$ & $-3.23 *$ & $-2.65^{*}$ & 2.26 & 2.18 \\
\hline P7XP9 & -0.12 & -0.97 & $6.09 * * *$ & $4.57 * *$ & $3.69 * * *$ & $4.08 * * *$ & 0.33 & 0.12 & -1.15 & -1.84 \\
\hline P7XP10 & $-2.10 * * *$ & $-3.28 * * *$ & $-7.47 * * *$ & $-5.83 * * *$ & $-8.25 * * *$ & $-9.32 * * *$ & $-7.88^{* * * *}$ & $-7.64 * * *$ & $2.59 *$ & 2.50 \\
\hline P8XP9 & 0.45 & 0.14 & 0.44 & -0.18 & -0.92 & -0.25 & $2.76^{*}$ & $2.91 *$ & $-2.64 *$ & $-3.17 *$ \\
\hline P8XP10 & -0.77 & -0.32 & -1.78 & 0.09 & $-5.44 * * *$ & $-5.10 * * *$ & $-5.24 * * * *$ & $-5.28 * * *$ & 1.78 & 1.68 \\
\hline P9XP10 & $-1.70 * *$ & $-1.87 *$ & 3.60 & 1.96 & $4.52 * * *$ & $5.33 * * *$ & 1.85 & $2.57^{*}$ & -0.19 & -0.43 \\
\hline \multicolumn{11}{|c|}{ C. D. Comparisons } \\
\hline Sij<>>0 at $95 \%$ & 1.15 & 1.73 & 2.76 & 2.70 & 1.51 & 1.29 & 2.58 & 2.35 & 2.54 & 2.68 \\
\hline $\mathrm{Sij}<>0$ at $99 \%$ & 1.54 & 2.31 & 3.68 & 3.61 & 2.02 & 1.72 & 3.44 & 3.14 & 3.40 & 3.57 \\
\hline Sij--Sik at $95 \%$ & 1.70 & 2.54 & 4.05 & 3.97 & 2.22 & 1.89 & 3.79 & 3.46 & 3.74 & 3.93 \\
\hline Sij--Sik at $99 \%$ & 2.26 & 3.39 & 5.41 & 5.30 & 2.97 & 2.53 & 5.06 & 4.62 & 5.00 & 5.25 \\
\hline Sij--Skl at 95\% & 1.62 & 2.42 & 3.86 & 3.78 & 2.12 & 1.80 & 3.61 & 3.29 & 3.57 & 3.75 \\
\hline Sij--Skl at 99\% & 2.16 & 3.23 & 5.16 & 5.05 & 2.83 & 2.41 & 4.82 & 4.40 & 4.76 & 5.01 \\
\hline
\end{tabular}


Table.6 Estimates of specific combining ability

\begin{tabular}{|c|c|c|c|c|c|c|c|c|c|c|c|c|}
\hline & \multicolumn{2}{|c|}{ Tillers/ Plant } & \multicolumn{2}{|c|}{ Spike Length } & \multicolumn{2}{|c|}{ Grains/ Spike } & \multicolumn{2}{|c|}{$\begin{array}{l}\text { 1000-grain Weight } \\
\text { (g) }\end{array}$} & \multicolumn{2}{|c|}{ Harvest Index (\%) } & \multicolumn{2}{|c|}{$\begin{array}{l}\text { Grain Yield/ Plant } \\
\text { (g) }\end{array}$} \\
\hline & $\mathrm{F}_{1}$ & $\mathrm{~F}_{2}$ & $\mathrm{~F}_{1}$ & $\mathrm{~F}_{2}$ & $\mathrm{~F}_{1}$ & $\mathrm{~F}_{2}$ & $\mathrm{~F}_{1}$ & $\mathrm{~F}_{2}$ & $\mathrm{~F}_{1}$ & $\mathrm{~F}_{2}$ & $\mathrm{~F}_{1}$ & $\mathrm{~F}_{2}$ \\
\hline P1XP2 & -0.70 & -0.28 & 0.19 & -0.44 & -1.01 & -1.93 & $4.14 * * *$ & $3.52 * *$ & 0.53 & 1.23 & 1.21 & 0.98 \\
\hline P1XP3 & 0.37 & 0.06 & -0.40 & $-1.51 *$ & -1.26 & -1.50 & $-2.19 *$ & -1.58 & -0.81 & -0.43 & -1.79 & -0.39 \\
\hline P1XP4 & 0.84 & 0.56 & $2.39 * *$ & $1.73 * *$ & $5.00 * * *$ & $4.27 * * *$ & -0.85 & -1.15 & -0.27 & 0.47 & $4.98 * * *$ & 0.64 \\
\hline P1XP5 & -0.56 & -0.16 & 0.09 & -0.77 & 0.08 & 0.09 & 1.60 & 1.10 & -1.52 & -1.51 & -1.05 & -1.03 \\
\hline P1XP6 & $1.20 *$ & 0.80 & $1.52 *$ & $2.15 * * *$ & $6.09 * * *$ & $6.43 * * *$ & 0.40 & -0.50 & $4.19 * * *$ & $5.65 * * *$ & $7.51 * * *$ & $3.74 * *$ \\
\hline P1XP7 & $-1.65 * *$ & $-1.82 * * *$ & -0.55 & $-1.48^{*}$ & $-5.82 * * *$ & $-6.88 * * *$ & $-3.43 * * *$ & $-3.99 * * *$ & $-5.63 * * *$ & $-7.62 * * *$ & -0.61 & -0.82 \\
\hline P1XP8 & 0.69 & -0.21 & $2.40 * *$ & 0.96 & $4.90 * * *$ & $4.53 * * *$ & $3.49 * * *$ & $2.73 *$ & $3.72 * *$ & $3.69 * *$ & $6.42 * * *$ & $2.39 *$ \\
\hline P1XP9 & 0.00 & -0.16 & $-1.98 * *$ & $-1.40^{*}$ & 2.66 & $2.89 * *$ & $-4.79 * * *$ & $-5.16 * * *$ & $-3.43 * *$ & $-5.43 * * *$ & $-4.12 * * *$ & $-4.34 * * *$ \\
\hline P1XP10 & -0.50 & -0.47 & -1.13 & -0.29 & 0.14 & $2.82 *$ & 1.29 & 0.54 & -1.06 & $-3.36 * *$ & -0.36 & -2.03 \\
\hline P2XP3 & -0.65 & -1.01 & $3.21 * * *$ & $1.47 *$ & $5.12 * * *$ & $5.31 * * *$ & $3.57 * * *$ & $2.93 *$ & $5.16 * * *$ & $5.65 * * *$ & $9.57 * * *$ & $2.22 *$ \\
\hline P2XP4 & 0.53 & 0.25 & $1.62 *$ & 1.15 & $7.48 * * *$ & $6.97 * * *$ & -0.99 & -2.14 & -0.68 & -0.36 & 1.27 & 0.69 \\
\hline P2XP5 & -0.46 & -0.71 & -1.00 & -1.11 & 0.16 & -1.41 & 1.11 & 0.05 & -1.81 & $-4.06 * * *$ & -0.10 & -0.95 \\
\hline P2XP6 & 0.04 & -0.77 & -0.13 & 0.63 & $-3.69 *$ & $-2.86^{*}$ & 0.88 & 0.10 & -0.87 & -0.24 & 0.40 & 0.76 \\
\hline P2XP7 & -1.11 & $-1.30 *$ & -0.29 & -0.53 & -2.75 & $-3.18 * *$ & $-3.25 * *$ & $-3.63 * *$ & -2.03 & $-2.30 *$ & $-2.55^{*}$ & -1.41 \\
\hline P2XP8 & -0.09 & 0.05 & -1.36 & 0.04 & -2.74 & $-3.17 * *$ & $-5.61 * * *$ & $-4.99 * * *$ & $-3.63 * *$ & $-3.85 * * *$ & $-3.74 * * *$ & -1.09 \\
\hline P2XP9 & 0.74 & $1.09 *$ & $1.59 *$ & $1.33 *$ & $3.50 *$ & $4.60 * * *$ & $4.38 * * *$ & $3.63 * *$ & 0.00 & $-6.50 * * *$ & $3.06 * *$ & 0.94 \\
\hline P2XP10 & 0.64 & 0.08 & 0.05 & -0.15 & -0.98 & -0.83 & $-2.79 * *$ & $-3.26 * *$ & -1.96 & 0.54 & -1.28 & -0.45 \\
\hline P3XP4 & 0.48 & 0.53 & $-1.74 *$ & -1.08 & $-3.06^{*}$ & $-2.32 *$ & 0.53 & -0.50 & $2.34^{*}$ & $-5.46 * * *$ & 1.26 & -1.91 \\
\hline P3XP5 & -1.06 & -0.90 & -0.96 & -0.45 & $-9.68 * * *$ & $-12.45 * * *$ & $-5.05 * * *$ & $-5.21 * * *$ & $-3.96 * * *$ & $3.26 * *$ & $-5.45 * * *$ & $-2.80^{*}$ \\
\hline P3XP6 & $1.46^{*}$ & 0.97 & $2.33 * *$ & $1.51 *$ & $5.17 * * *$ & $6.19 * * *$ & $-1.97 *$ & $-2.90^{*}$ & $2.87 *$ & $-4.39 * * *$ & $6.00 * * *$ & $3.63 * *$ \\
\hline P3XP7 & -0.51 & -0.95 & 1.07 & -0.23 & $4.24 * *$ & $4.44 * * *$ & $-3.85 * * *$ & $-4.95 * * *$ & $-2.85^{*}$ & 0.33 & -1.82 & -0.66 \\
\hline P3XP8 & -0.49 & -0.16 & 0.27 & 0.57 & $4.55 * *$ & $4.06^{* * * *}$ & 1.62 & 0.43 & -1.25 & -2.00 & $2.94 * *$ & 0.89 \\
\hline P3XP9 & 0.31 & 0.03 & -0.49 & -0.19 & $4.87 * * *$ & $5.45 * * *$ & $3.98 * * *$ & $3.97 * * *$ & -0.87 & -1.34 & 1.11 & -1.60 \\
\hline P3XP10 & 0.49 & 0.13 & -0.38 & 0.57 & -1.89 & $-3.20 * *$ & 0.67 & -0.02 & -1.99 & -0.09 & -0.60 & -0.50 \\
\hline P4XP5 & $-1.35^{*}$ & $-1.56 * *$ & $-2.09 * *$ & $-2.17 * * *$ & $-6.62 * * *$ & $-7.75 * * *$ & $-5.80 * * *$ & $-5.71 * * *$ & $-6.89 * * *$ & $-4.82 * * *$ & $-7.41 * * *$ & $-5.27 * * *$ \\
\hline P4XP6 & -0.87 & -0.63 & -0.92 & 0.41 & 2.72 & $3.45 * *$ & -0.85 & -1.64 & $2.40 *$ & $-5.75 * * *$ & $3.69 * * *$ & 0.90 \\
\hline P4XP7 & $1.24 *$ & 0.86 & 0.71 & 0.20 & $6.44 * * *$ & $5.95 * * *$ & $3.05 * *$ & $2.74 *$ & $2.41 *$ & 1.83 & $4.78 * * *$ & 0.67 \\
\hline P4XP8 & 0.11 & -0.27 & 0.92 & 1.06 & -1.13 & -0.72 & $2.62 * *$ & $3.22 * *$ & -0.42 & $6.67 * * *$ & $2.04 *$ & 0.95 \\
\hline P4XP9 & $-1.45^{*}$ & $-1.45^{* *}$ & -1.35 & $-2.42 * * *$ & $-5.19 * * *$ & $-4.53 * * *$ & -1.38 & $-2.23^{*}$ & $-4.18 * * *$ & 1.57 & $-6.52 * * *$ & $-4.09 * * *$ \\
\hline P4XP10 & -0.17 & -0.45 & 0.18 & -0.68 & -1.14 & $-3.33 * *$ & 0.38 & -0.38 & -0.48 & $-3.13 * *$ & -1.39 & -1.69 \\
\hline P5XP6 & 0.89 & 0.60 & 0.07 & -0.28 & $-3.55^{*}$ & $-3.95 * * *$ & $2.46^{*}$ & $3.08 * *$ & 0.08 & 1.35 & 0.13 & -0.23 \\
\hline P5XP7 & 0.44 & 0.14 & $1.43^{*}$ & $1.74 * *$ & $5.28 * * *$ & $7.05 * * *$ & $3.16 * *$ & $3.16 * *$ & $3.97 * * *$ & -0.96 & $6.89 * * *$ & $3.28 * *$ \\
\hline P5XP8 & 0.09 & -0.25 & $2.21 * *$ & 0.55 & $5.58 * * *$ & $3.85 * * *$ & -0.79 & -2.06 & $2.58 *$ & $7.06 * * *$ & $5.62 * * *$ & $2.42 *$ \\
\hline P5XP9 & 0.59 & 0.41 & $2.81 * * *$ & $2.60 * * *$ & $7.19 * * *$ & $6.22 * * *$ & $2.84 * *$ & $3.54 * *$ & $8.14 * * *$ & 1.20 & $8.14 * * *$ & $3.35 * *$ \\
\hline P5XP10 & -0.70 & $-1.16^{*}$ & -0.90 & -0.88 & -1.36 & $9.27 * * *$ & 1.12 & -0.24 & -1.84 & -2.07 & $-2.38^{*}$ & -1.04 \\
\hline P6XP7 & -0.68 & -0.81 & $-1.88^{*}$ & $-1.72 * *$ & -0.98 & -1.00 & $2.45^{*}$ & $2.44 *$ & -1.48 & -0.72 & $2.36^{*}$ & -1.26 \\
\hline P6XP8 & -0.71 & -0.20 & -0.83 & $-1.54 *$ & $-5.90 * * *$ & $-5.37 * * *$ & $2.21 *$ & 2.00 & $-2.32 *$ & -0.66 & $-2.37^{*}$ & -0.59 \\
\hline P6XP9 & 0.12 & 0.19 & $1.78 *$ & -0.76 & $7.25 * * *$ & 0.37 & 0.83 & 0.00 & $3.70 * *$ & -1.41 & $5.91 * * *$ & 0.54 \\
\hline P6XP10 & -1.01 & $-1.07 *$ & -0.11 & $-1.26^{*}$ & 0.97 & -0.03 & $-3.57 * * *$ & $-3.48 * *$ & -2.11 & $4.61 * * *$ & $-3.06 * *$ & -0.67 \\
\hline P7XP8 & 0.35 & 0.17 & $1.96 * *$ & $2.26 * * *$ & $6.54 * * *$ & $7.98 * * *$ & 0.82 & 0.50 & $5.45 * * *$ & $-7.94 * * *$ & $6.66 * * *$ & $3.36 * *$ \\
\hline P7XP9 & $1.23 *$ & 0.86 & $-2.21 * *$ & $-2.33 * * *$ & $-8.48 * * *$ & $-8.36 * * *$ & $2.84 * *$ & 1.93 & $-3.47 * *$ & $8.06^{* * *}$ & $-4.44 * * *$ & $-3.18 * *$ \\
\hline P7XP10 & 0.23 & 0.66 & $2.46 * *$ & $4.23 * * *$ & $9.61 * * *$ & $8.05 * * *$ & $4.58 * * *$ & $3.60 * *$ & $4.30 * * *$ & $2.96 * *$ & $8.30 * * *$ & $5.20 * * *$ \\
\hline P8XP9 & -0.26 & $-1.05^{*}$ & -0.82 & -0.32 & -0.62 & -0.29 & $-3.11 * *$ & $-4.93 * * *$ & -1.83 & $2.44^{*}$ & -1.18 & -0.79 \\
\hline P8XP10 & 0.69 & 0.43 & $2.76 * * *$ & $1.58 *$ & $8.63 * * *$ & $7.09 * * *$ & $3.45 * * *$ & $3.32 * *$ & $4.34 * * *$ & 0.44 & $9.98 * * *$ & $4.87 * * *$ \\
\hline P9XP10 & -0.01 & -0.42 & 1.23 & -0.55 & -1.87 & $-2.71 *$ & 0.43 & 0.11 & 0.51 & -0.34 & 0.86 & -0.57 \\
\hline \multicolumn{13}{|c|}{ C. D. Comparisons } \\
\hline $\mathrm{Sij}<>0$ at $95 \%$ & 1.17 & 1.03 & 1.43 & 1.20 & 2.77 & 2.16 & 1.95 & 2.20 & 2.25 & 2.18 & 1.95 & 2.21 \\
\hline $\mathrm{Sij}<>0$ at $99 \%$ & 1.56 & 1.38 & 1.91 & 1.60 & 3.70 & 2.89 & 2.61 & 2.94 & 3.00 & 2.91 & 2.61 & 2.96 \\
\hline Sij--Sik at $95 \%$ & 1.72 & 1.52 & 2.10 & 1.77 & 4.08 & 3.18 & 2.87 & 3.23 & 3.30 & 3.20 & 2.87 & 3.25 \\
\hline Sij--Sik at $99 \%$ & 2.30 & 2.03 & 2.80 & 2.36 & 5.45 & 4.24 & 3.83 & 4.32 & 4.41 & 4.28 & 3.83 & 4.34 \\
\hline Sij--Skl at $95 \%$ & 1.64 & 1.45 & 2.00 & 1.68 & 3.89 & 3.03 & 2.73 & 3.08 & 3.15 & 3.05 & 2.73 & 3.10 \\
\hline
\end{tabular}


Int.J.Curr.Microbiol.App.Sci (2018) 7(7): 1586-1593 


\section{References}

Anonymous, 2015. Population growth (annual $\%$ ). World Bank. Retrieved 20 January 2015.

Adel, M.M. and Ali, E.A. 2013. Gene action and combining ability in a six parent diallel cross of wheat. Asian J. Crop Sci., 5 (1): 14-23.

Bhardwaj, R Genetic Studies for Yield and its Contributing Attributes in Bread Wheat (Triticum aestivum L. em Thell) Over Environments. Thesis (Unpub.) submitted to S. K. N. A. U. Jobner.

Grafius, J.E. 1959. Heterosis in barley. Agron. J., 51 (9): 551-554.

Grifing, B. 1956. Concept of general and specific combining ability in relation to diallel crossing system. Aust. J. Biol. Sci., 9 (4): 463-493.

Hull, F.H. 1945. Recurrent selection for specific combining ability in corn. J. Amer. Soc. Agron., 37: 134-145.

Irshad, M.; Khaliq,I.; Iqbal, J.; Hussain, N.; Aslam, M.; Hussain, K. and Din,N. 2014. Genetics of some polygenic traits in hexaploid bread wheat in high temperature stress. J. Animal and pl br. Sci., 24 (4): 1212-1219.

Jinks, J.L. and Jones, R.M. 1958. Estimation of components of heterosis. Genet., 43 (2): 223-234.

Kalhoro, F.A., Rajpar, A.A., Kalhoro, S.A., Mahar, A., Ali, A., Otho, S.A., Soomro, R.N., Ali, F. and Baloch, Z.A. (2015). Heterosis and combing ability in F1 population of hexaploid wheat (Triticum Aestivum L.). Am. J. Pl. Sci., 6: 1011-1026.

Kaukab, S., Saeed, M.S. and Rehman, A.U. 2014. Genetic analysis for yield and some yield traits in spring wheat. Uni. J. Agric. Res., 2 (7): 272-277.

Kumar, D. and Kerkhi, S.A. 2015.Combining ability analysis for yield and some quality traits in spring wheat ( $\mathrm{T}$. aestivum L.). Electronic J. of Pl. Br., 6 (1): 26.

Mari, S.N.; Ansari, B.A.; Kumbhar, M.B. and Keerio, M.I. 2015. Gene action governing inheritance of economically valued traits among F1 hybrids of hexaploid wheat derived through diallel matting system. Sindh Univ. Res. J., 47 (4): 663-668.

Moll, R.H., Lindsey, M.F. and Robinson, H.F. 1964. Estimates of genetic variances and level of dominance in maize. Genet., 49: 411-423.

Samir, K.A.; Ismail. 2015. Heterosis and combining ability analysis for yield and it components in bread wheat (Triticum aestivum L.). Int. J. Curr. Microbio. App. Sci., 4 (8): 1-9.

Sharma, J.K. and Singh, H.B. 1990. Combining ability in bread wheat (Triticum aestivum L.). Himachal J. Agril. Res., 16 (1/2): 48-55.

Yadav, J. 2017. Genetic architecture of yield and its associated traits in bread wheat (Triticum aestivum L. em. Thell.) over environments. Thesis (Unpub.) submitted to S. K. N. A. U. Jobner.

\section{How to cite this article:}

Ritu Choudhary, Hoshiyar Singh, Pawan Kumar and Sheetal Raj Sharma. 2018. General and Specific Combining Ability Analysis for Yield and Some of Its Associated Characters in Late Sown Condition in Bread Wheat (Triticum aestivum L.em. Thell). Int.J.Curr.Microbiol.App.Sci. 7(07): 1586-1593. doi: https://doi.org/10.20546/ijcmas.2018.707.187 